Article

\title{
Sexually Dimorphic Behavioral Profile in a Transgenic Model Enabling Targeted Recombination in Active Neurons in Response to Ketamine and (2R,6R)-Hydroxynorketamine Administration
}

\author{
David P. Herzog 1,+(1D, Ratnadevi M. Mellema ${ }^{1,+}$, Floortje Remmers ${ }^{2} \mathbb{D}^{\mathbb{D}}$, Beat Lutz ${ }^{2,3}$, \\ Marianne B. Müller $1,3, \ddagger$ and Giulia Treccani $1,3,4, *, \ddagger$ \\ 1 Laboratory of Translational Psychiatry and Focus Program Translational Neurosciences, Johannes Gutenberg \\ University Medical Center Mainz, 55128 Mainz, Germany; daherzog@uni-mainz.de (D.P.H.); \\ ratna_mellema@msn.com (R.M.M.); marianne.mueller@uni-mainz.de (M.B.M.) \\ 2 Institute of Physiological Chemistry, Johannes Gutenberg University Medical Center Mainz, \\ 55128 Mainz, Germany; remmersf@uni-mainz.de (F.R.); beat.lutz@uni-mainz.de (B.L.) \\ 3 Leibniz Institute for Resilience Research, 55131 Mainz, Germany \\ 4 Translational Neuropsychiatry Unit, Department of Clinical Medicine, Aarhus University, \\ 8000 Aarhus, Denmark \\ * Correspondence: gtreccan@uni-mainz.de; Tel.: +49-(0)6131-39-21345 \\ + These authors contribute equally to this work. \\ $\ddagger \quad$ These authors shared last authorship.
}

Received: 31 January 2020; Accepted: 17 March 2020; Published: 20 March 2020

\begin{abstract}
Background: Rapid-acting antidepressants ketamine and (2R,6R)-hydroxynorketamine $((2 \mathrm{R}, 6 \mathrm{R})-\mathrm{HNK})$ have overcome some of the major limitations of classical antidepressants. However, little is known about sex-specific differences in the behavioral and molecular effects of ketamine and $(2 \mathrm{R}, 6 \mathrm{R})-\mathrm{HNK}$ in rodents. Methods: We treated mice with an intraperitoneal injection of either saline, ketamine $\left(30 \mathrm{mg} \mathrm{kg}^{-1}\right)$ or $(2 \mathrm{R}, 6 \mathrm{R})-\mathrm{HNK}\left(10 \mathrm{mg} \mathrm{kg}^{-1}\right)$. We performed a comprehensive behavioral test battery to characterize the Arc-CreERT2 $\times$ CAG-Sun1/sfGFP mouse line which enables targeted recombination in active populations. We performed a molecular study in Arc-CreERT2 $\times$ CAG-Sun1/sfGFP female mice using both immunohistochemistry and in situ hybridization. Results: Arc-CreERT2 $\times$ CAG-Sun1/sfGFP mice showed sex differences in sociability and anxiety tests. Moreover, ketamine and (2R,6R)-HNK had opposite effects in the forced swim test (FST) depending on gender. In addition, in male mice, ketamine-treated animals were less immobile compared to $(2 \mathrm{R}, 6 \mathrm{R})-\mathrm{HNK}$, thus showing a different profile of the two drugs in the FST. At the molecular level we identified $B d n f$ mRNA level to be increased after ketamine treatment in female mice. Conclusion: Arc-CreERT2 $\times$ CAG-Sun1/sfGFP mice showed sex differences in social and anxiety behavior and a different pattern between ketamine and (2R,6R)-HNK in the FST in male and female mice. At the molecular level, female mice treated with ketamine showed an increase of Bdnf mRNA level, as previously observed in male mice.
\end{abstract}

Keywords: ketamine; hydroxynorketamine; antidepressant; rapid-acting; sex difference; BDNF; activated neurons; behavior

\section{Introduction}

Major depressive disorder (MDD) poses a serious threat on modern societies [1] and novel treatment approaches besides classical antidepressants are needed to advance treatment of this devastating illness. In recent decades, the emergence of rapid-acting antidepressants in preclinical and 
clinical studies have overcome some of the limitations of MDD therapy: the effect latency [2] and the high rate of non-response [3] to most classical antidepressants.

The rapid-acting antidepressant ketamine has been repeatedly reported to be effective in both animal models of MDD [3] and patients [4]: in contrast to classical antidepressants, ketamine exerted its effects within hours [5], lasting for several days [5], and was even found effective in patients with treatment-resistant MDD [5]. However, ketamine treatment comes with some relevant side effects like nausea, sedation, and the risk of addiction, which so far prevented its wide-spread use [6]. So far, ketamine administration has had to be via parenteral administration, further impairing its applicability in the clinic. However, just recently a new ketamine nasal spray in combination with an oral antidepressant has been approved by the FDA for treatment-resistant depression, hence providing a new available formulation [7]. To avoid the ketamine side-effects, researchers also looked into the various ketamine metabolites, lacking these side-effects. Zanos and colleagues recently showed that (2R,6R)-hydroxynorketamine $((2 \mathrm{R}, 6 \mathrm{R})$-HNK) is a ketamine metabolite with similar antidepressant-like efficacy in rodents, but without the ketamine-related side effects [8].

The antidepressant ketamine in mice affects several behavioral domains. It was found to be anxiolytic [9] as well as to reduce the immobility time in the forced swim test (FST), a commonly used test for depressive-like behavior [10]. However, long-term administration of ketamine seemed to impair cognitive function in mice [11]. Many behavioral studies observed a significant difference in the behavioral effects of ketamine based on gender. Indeed, female mice were reported to be more susceptible to the effects of ketamine: they respond to lower doses of ketamine [12] and exhibit more pronounced acute and long-term antidepressant-like effects [13]. These differences might be explained by sex-specific alterations of hormones and metabolism: estrogen receptor agonists lowered the dose required to produce an antidepressant-like effect in female mice [12] and female rodents developed higher plasma and brain ketamine concentrations, followed by a slower elimination of the substance from the organism [14]. Studies addressing the role of gender in (2R,6R)-HNK treatment are limited. Published work so far did not detect a role of gender in the behavioral response to (2R,6R)-HNK administration. Recently, we summarized the evidence of the role of sex in the effects of ketamine and (2R,6R)-HNK [15].

The molecular mechanisms of action of ketamine and (2R,6R)-HNK are only partly understood: there are key neurobiological mechanisms that are well-known factors of MDD pathology and therapy, which were found to play a role in the rapid-acting effects of ketamine. For example, a single injection of ketamine activated the mammalian target of rapamycin (mTOR) and this enhanced synaptogenesis in the medial prefrontal cortex (mPFC) [16], reversing the stress-induced synaptic deficits in mice [17]. Ketamine administration led to the fast production of brain neurotrophic factor (BDNF) [18], an important mediator of MDD pathology and treatment [19], by inducing glutamate bursts and activating postsynaptic $\alpha$-amino-3-hydroxy-5-methyl-4-isoxazolepropionic acid receptor (AMPA) receptors [20]. Similar to ketamine, in vitro [21] and in vivo [22] experiments showed that (2R,6R)-HNK induced structural and synaptic plasticity. A similar involvement of the mTOR pathway and BDNF in the mechanisms of action of (2R,6R)-HNK is likely. Just recently, Fukumoto et al. showed that BDNF signaling is required for the antidepressant-like effects of $(2 \mathrm{R}, 6 \mathrm{R})-\mathrm{HNK}$ [23].

The behavioral and molecular effects of ketamine and $(2 \mathrm{R}, 6 \mathrm{R})-\mathrm{HNK}$ were in the focus of the present study. We analyzed these effects using a transgenic mouse line capable of a targeted recombination in active populations (TRAP), the Arc-CreERT2 $\times$ CAG-Sun1/sfGFP [24]. This mouse line allows the permanent labeling of activated neurons by combining a green fluorescent protein (GFP) conditional reporter line, and a tamoxifen (TM)-dependent Cre-line wherein the expression of Cre is under the control of an immediate early gene promoter, in this case under the activity-regulated cytoskeleton-associated protein (Arc) promoter [25]. Ketamine is known to enhance Arc expression [25] and hence in this line a GFP signal is produced in any neuron with a rise in Arc expression [24]. So far, comprehensive and sex-specific behavioral characterization of the effects of ketamine and HNK on the Arc-CreERT2 $\times$ CAG-Sun1/sfGFP mouse line is lacking. First, we wanted to fill this gap of knowledge by applying a condensed behavioral 
battery to assess female and male Arc-CreERT2 $\times$ CAG-Sun1/sfGFP mice in several behavioral domains. In a second step, we performed a molecular analysis elucidating the effects of ketamine and (2R,6R)-HNK on Bdnf expression using Arc-CreERT2 × CAG-Sun1/sfGFP female mice.

\section{Results}

\subsection{Behavioral Characterization}

Arc-CreER ${ }^{\mathrm{T} 2} \times$ CAG-Sun1/sfGFP mice were subjected to a test battery that enabled characterization of multiple behavioral domains in a limited amount of time [26]. These experiments were designed in order to characterize the behavioral phenotype of female and male mice in the absence of (tamoxifen induced) recombination and yet to understand which are the behavior changes in response to ketamine and $(2 \mathrm{R}, 6 \mathrm{R})-\mathrm{HNK}$ treatment.

\subsubsection{Ketamine and (2R,6R)-HNK Do not Affect Spatial (Working) or Episodic Memory}

Statistical analysis of the spontaneous alternations, spatial object recognition task (SORT) and novel object recognition (NORT) task demonstrated no effect of treatment (Figure 1A-C, Table 1), suggesting that ketamine and $(2 \mathrm{R}, 6 \mathrm{R})-\mathrm{HNK}$ did not affect hippocampal-dependent memory performance $24 \mathrm{~h}$ after administration. In the spontaneous alternation test all treatment groups revealed around $60 \%$ spontaneous alternation performance (SAP) behavior, indicating intact working memory (Figure 1A, Tables 1 and 2 for detailed statistics). In the SORT test, only female mice showed significant spatial object recognition above chance level (50\% cut off), whereas males did not (Figure 1B, Tables 1 and 2). In the NORT, saline-treated, but neither ketamine nor (2R,6R)-HNK treated females showed significant novel object recognition above chance level (Figure 1C, Tables 1 and 2).
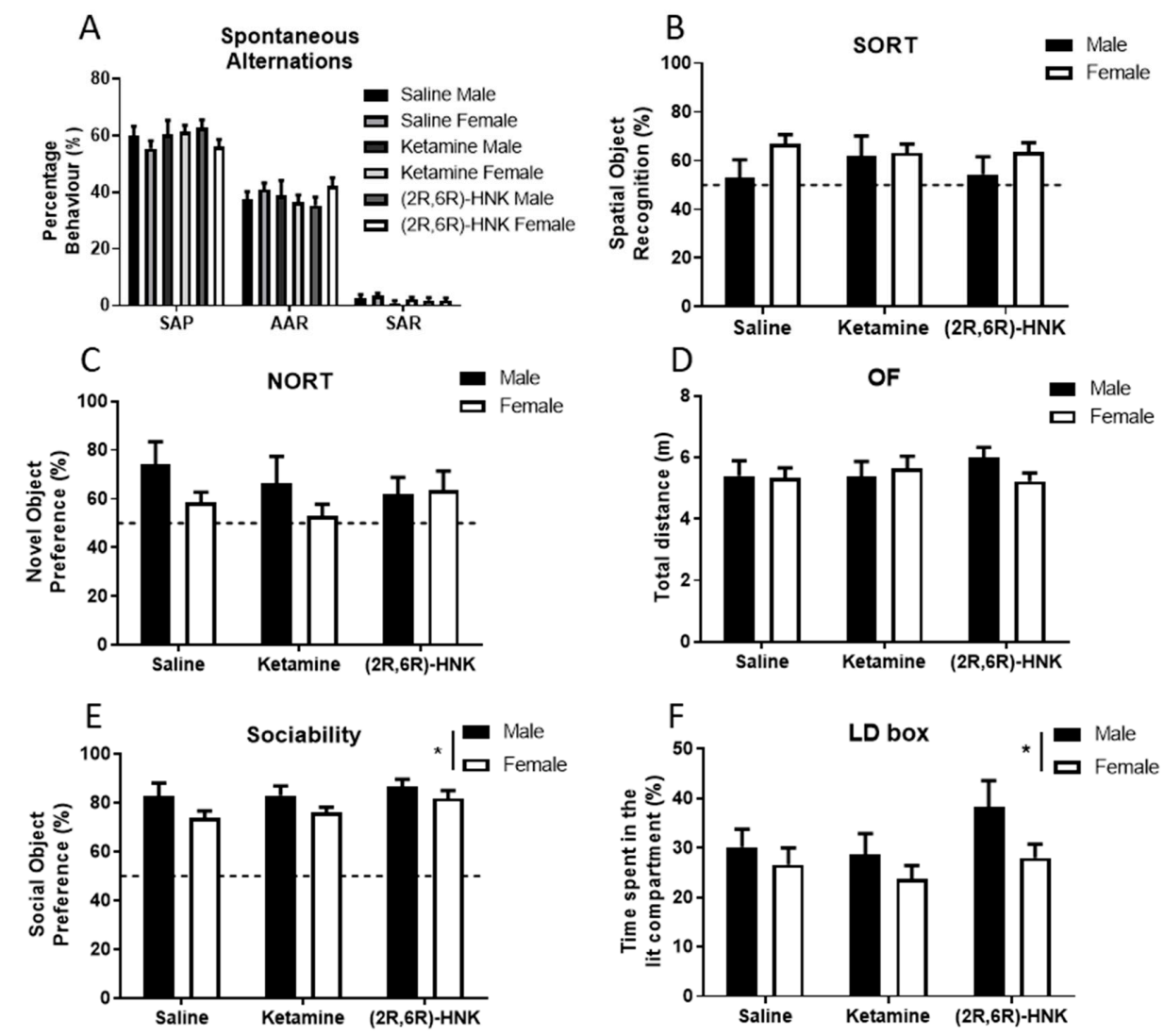

Figure 1. Sex, but not treatment with ketamine or (2R,6R)-hydroxynorketamine ((2R,6R)-HNK) affected sociability and anxiety of Arc-CreER ${ }^{\mathrm{T} 2} \times$ CAG-Sun1/sfGFP mice $24 \mathrm{~h}$ post-injection. (A) Spontaneous alternations in the Y-maze. The frequency of the behaviors spontaneous alternation performance (SAP), 
alternate arm returns (AAR) and same arm returns (SAR) in treatment groups was counted and is presented in percentages. Male $n=21$, female $n=39$. (B) Spatial object recognition task (SORT). Exploration time of the displaced object was measured (s), spatial object recognition was calculated as and is presented in percentage. Male $n=21$, female $n=39$. (C) Novel object recognition task (NORT). Novel object interaction time was measured (s) and presented in percentage of the total exploration time. Male $n=16$, female $n=30$. (D) Locomotor activity in the open field (OF). Total distance was calculated in meters. Male $n=22$, female $n=39$. (E) Sociability test. Preference for the social object (juvenile male mouse) was measured and is presented in percentage. Sex difference was observed in the preference for the social object ( $p=0.0133$, for detailed statistics see Table 1 ). Male $n=21$, female $n=37$. (F) Anxiety-like behavior in the light-dark box (LD box). Time spent in the lit compartment was calculated and presented in percentage. Sex difference was observed in the time spent in the lit compartment ( $p=0.0405$, for detailed statistics see Table 1 ). Male $n=22$, female $n=39$. All behaviors are expressed as mean and error bars represent SEM, dotted line represents chance level, ${ }^{*} p<0.05$.

Table 1. Statistical analysis presenting the effect of treatment and sex on the performance in different behavioral tests (two-way ANOVA). $\mathrm{F}=\mathrm{f}$-distribution, $\mathrm{dfn}=$ degrees of freedom numerator, $\mathrm{dfd}=$ degrees of freedom denominator. ${ }^{*}$ Bold $p$ values represent significant values.

\begin{tabular}{ccccc}
\hline Behavioural Test & Effect & $p$ Value & F & dfn, dfd \\
\hline \multirow{2}{*}{ Spontaneous } & Treatment & 0.7364 & 0.3077 & 2,56 \\
alternations & Sex & 0.8434 & 0.0394 & 1,56 \\
& Interaction & 0.0816 & 2.621 & 2,56 \\
\hline \multirow{2}{*}{ SORT } & Treatment & 0.7892 & 0.2377 & 2,54 \\
& Sex & 0.0678 & 3.475 & 1,54 \\
& Interaction & 0.5034 & 0.6952 & 2,54 \\
\hline \multirow{2}{*}{ NORT } & Treatment & 0.6674 & 0.4085 & 2,40 \\
& Sex & 0.1274 & 2.423 & 1,40 \\
& Interaction & 0.4187 & 0.8899 & 2,40 \\
\hline \multirow{3}{*}{ OF } & Treatment & 0.8226 & 0.196 & 2,55 \\
& Sex & 0.5454 & 0.3703 & 1,55 \\
& Interaction & 0.4192 & 0.8833 & 2,55 \\
\hline \multirow{2}{*}{ Sociability } & Treatment & 0.1729 & 1.816 & 2,52 \\
& Sex & $\mathbf{0 . 0 1 3 3}$ * & 6.574 & 1,52 \\
& Interaction & 0.8376 & 0.1779 & 2,52 \\
\hline \multirow{2}{*}{ LD box } & Treatment & 0.1593 & 1.9 & 2,55 \\
& Sex & $\mathbf{0 . 0 4 0 3 *}$ & 4.411 & 1,55 \\
& Interaction & 0.6075 & 0.503 & 2,55 \\
\hline
\end{tabular}

Table 2. Statistical analysis of the performance in the behavioral battery compared to chance level (one sample $t$-test). $t=t$ score, $\mathrm{df}=$ degrees of freedom. Bold $p$ values represent significant values, indicating difference compared to $50 \%$ chance level.

\begin{tabular}{|c|c|c|c|c|c|c|c|}
\hline \multirow{2}{*}{ Behavioural Test } & \multirow{2}{*}{ Treatment } & \multicolumn{3}{|c|}{ Male } & \multicolumn{3}{|c|}{ Female } \\
\hline & & $p$ Value & $t$ & df & $p$ Value & $t$ & df \\
\hline \multirow{3}{*}{ SORT } & Saline & 0.694 & 0.417 & 5 & 0.0003 & 4.851 & 13 \\
\hline & Ketamine & 0.2043 & 1.424 & 6 & 0.0029 & 3.65 & 13 \\
\hline & HNK & 0.5703 & 0.5954 & 7 & 0.0034 & 3.717 & 11 \\
\hline \multirow{3}{*}{ NORT } & Saline & 0.0817 & 2.581 & 3 & 0.0412 & 2.311 & 11 \\
\hline & Ketamine & 0.216 & 1.468 & 4 & 0.5445 & 0.6274 & 10 \\
\hline & HNK & 0.149 & 1.655 & 6 & 0.1432 & 1.649 & 7 \\
\hline \multirow{3}{*}{ Sociability } & Saline & 0.0016 & 6.228 & 5 & $<0.0001$ & 8.531 & 12 \\
\hline & Ketamine & 0.0001 & 8.657 & 6 & $<0.0001$ & 11.96 & 12 \\
\hline & HNK & $<0.0001$ & 13.02 & 7 & $<0.0001$ & 9.878 & 11 \\
\hline \multirow{3}{*}{$\begin{array}{l}\text { Bedding } \\
\text { Preference }\end{array}$} & Saline & 0.0952 & 1.978 & 6 & 0.1845 & 1.402 & 13 \\
\hline & Ketamine & 0.0339 & 2.736 & 6 & 0.2512 & 1.201 & 13 \\
\hline & HNK & $<0.0001$ & 11.4 & 7 & 0.3102 & 1.064 & 11 \\
\hline
\end{tabular}




\subsubsection{Sex, but Not Treatment, Affected Social Behavior and Anxiety}

Other behavioral domains that were examined in the behavioral battery comprised locomotion, sociability preference, and anxiety-like behavior. Statistical analysis of performance in the open field $(\mathrm{OF})$ revealed that ketamine and $(2 \mathrm{R}, 6 \mathrm{R})-\mathrm{HNK}$ did not affect the total distance mice travelled (Figure 1D, Table 1). All treatment groups travelled an average distance of $5.5 \mathrm{~m}$ in the OF, independent of sex. Statistical analysis of the sociability test showed that ketamine and (2R,6R)-HNK did not affect sociability (Table 1, Figure 1E). All treatment groups showed significant preference for the social object compared to chance level (see Tables 1 and 2 for statistics), suggesting intact social behavior. In contrast, sex had a significant main effect on sociability (see Table 1 for statistics). Likewise, anxiety-like behavior, measured as avoidance of the lit compartment of the light-dark (LD) box (Figure 1F) was significantly affected by sex (Table 1). Importantly, statistical analysis showed that anxiety-like behavior tested in the LD box was not affected by ketamine nor (2R,6R)-HNK (Table 1). Neither treatment nor sex affected olfaction in the bedding preference test (Figure A1 and Table 1).

2.1.3. Sex Influenced the Antidepressant-Like Effects of Ketamine and (2R,6R)-HNK in the Forced Swim Test (FST)

To assess the antidepressant-like effect of ketamine and (2R,6R)-HNK in the Arc-CreER ${ }^{\mathrm{T} 2} \times$ CAG-Sun1/sfGFP mouse line, male and female mice were subjected to the forced swim test $24 \mathrm{~h}$ after a single injection with either saline, ketamine, or $(2 \mathrm{R}, 6 \mathrm{R})-\mathrm{HNK}$. Statistical analysis determined a significant interaction: the effects of saline, ketamine, or $(2 R, 6 R)-H N K$ were opposite in male and female mice (Figure 2) with males more immobile after saline and (2R,6R)-HNK treatment and less immobile after ketamine treatment, while female showed the opposite in each condition. We also detected a statistical trend for sex difference. Post-test analysis also revealed that ketamine treated male mice were less immobile than those treated with $(2 \mathrm{R}, 6 \mathrm{R})-\mathrm{HNK}$, hence suggesting that ketamine and $(2 \mathrm{R}, 6 \mathrm{R})-\mathrm{HNK}$ revealed different effects in the FST (Figure 2).

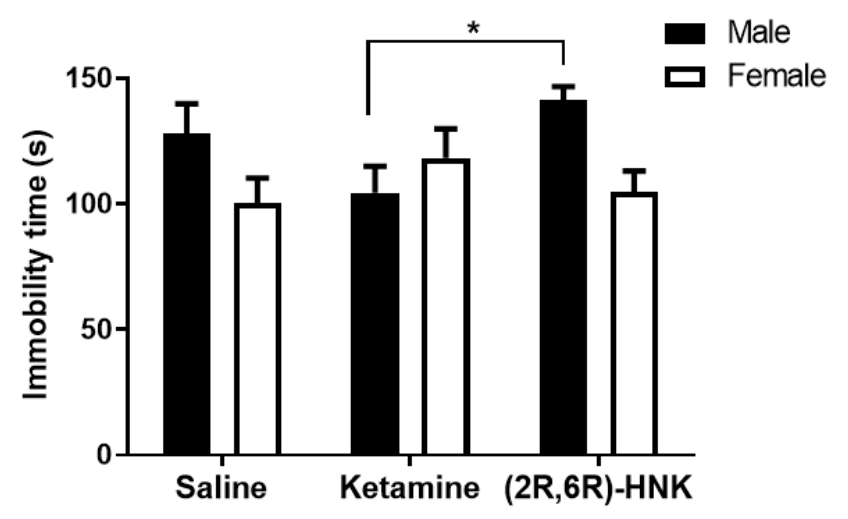

Figure 2. Effects on the immobility time measured in the forced swim test (FST) $24 \mathrm{~h}$ after a single injection with ketamine or (2R,6R)-HNK in Arc-CreER ${ }^{\mathrm{T} 2} \times$ CAG-Sun1/sfGFP mice. (Two-way ANOVA: interaction: $\mathrm{F}(2,80)=3.397, p=0.0384$; sex: $\mathrm{F}(1,80)=3.924, p=0.0510 ; n=11-18$ mice per group). Bonferroni post test showed that male mice treated with ketamine are more immobile than those treated with $(2 \mathrm{R}, 6 \mathrm{R})$-HNK $[t=2.89 \mathrm{df}=80, * p<0.05]$. Immobility time is presented in mean (s), error bars represent \pm SEM.

\subsection{Molecular Characterization}

A key component in the mechanism of action of ketamine is the activation of neuroplasticity mechanism and among those, the increase of BDNF [18]. Surprisingly, BDNF increase has been mainly reported in male mice. Hence, it is of interest to confirm that a similar mechanism is taking place in female mice. By taking advantage of the use of the Arc-CreER ${ }^{\mathrm{T} 2} \times \mathrm{CAG}_{\mathrm{S}} \mathrm{Sun} 1 / \mathrm{sfGFP}$ mice, we identified molecular changes only occurring in activated neurons, wherein tamoxifen-induced 
targeted recombination took place in response to stimuli, in our case ketamine, $(2 \mathrm{R}, 6 \mathrm{R})-\mathrm{HNK}$, or saline treatment.

\subsubsection{Activated Nuclei Upon Ketamine and (2R,6R)-HNK Administration}

In the Arc-CreERT2 $\times$ CAG-Sun1/sfGFP mice, TM administration causes active (Arc-expressing and therefore) CreERT2-expressing cells to undergo Cre-mediated recombination (to be "TRAPed"), resulting in permanent expression of the effector gene (GFP; Figure 3A-C). Nonactive cells do not express CreERT2 and do not undergo recombination, even in the presence of TM. We therefore quantified the mean intensity of GFP labelled activated nuclei of female mice. Statistical analysis did not show any significant differences between treatment groups in neither the dentate gyrus (DG) nor the Cornu Ammonis region 3 (CA3) (Figure 3D,E). However, a strong trend towards significance was evident in the $\mathrm{CA} 3$ region of the hippocampus.

\section{A}
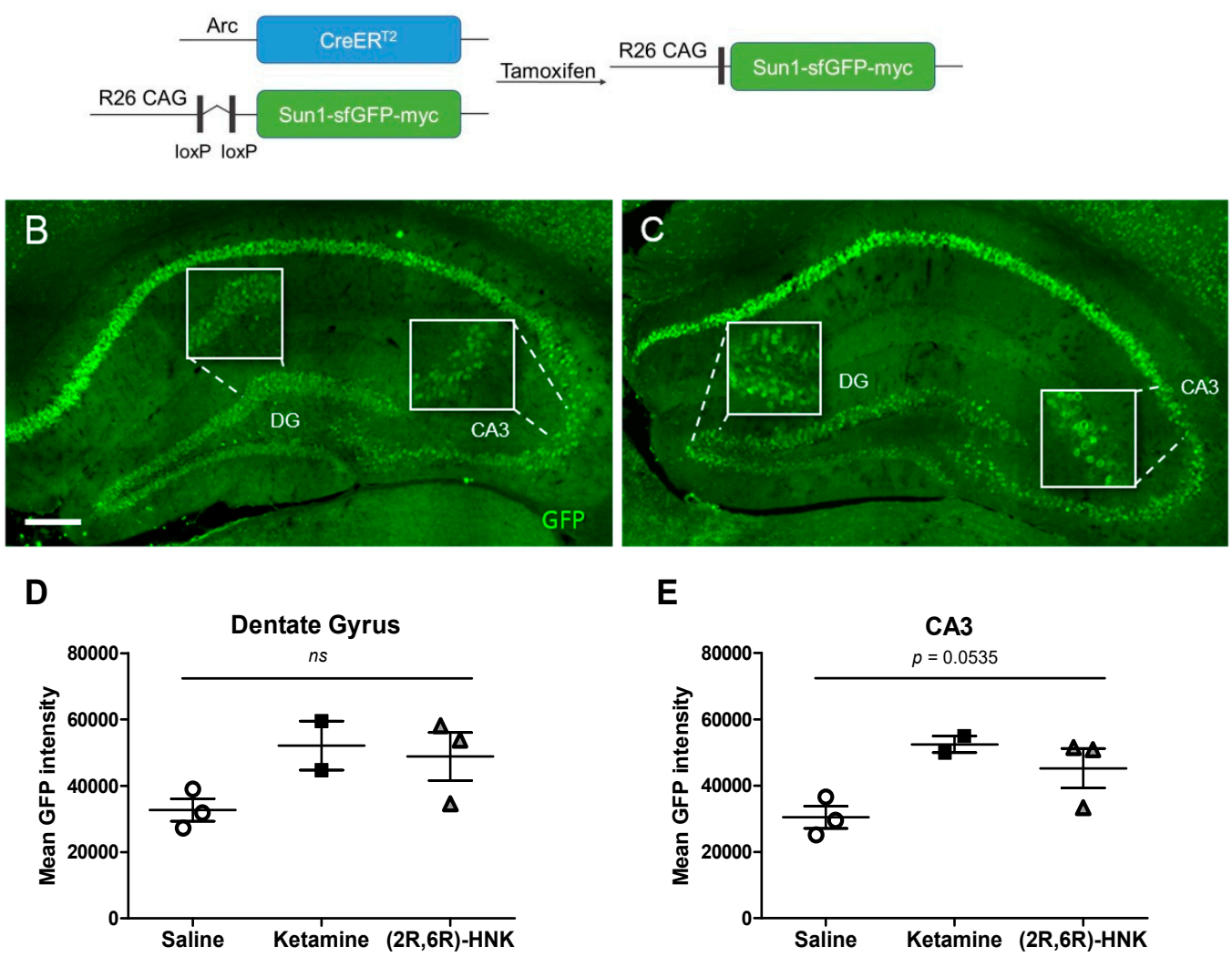

Figure 3. Green fluorescent protein (GFP)-labelled activated nuclei in the hippocampal dentate gyrus (DG) and Cornu Ammonis region 3 (CA3) after a single injection with ketamine or (2R,6R)-HNK in female mice. (A) Genetic design. Administration of tamoxifen (TM) to Arc-CreER ${ }^{\mathrm{T} 2} \times \mathrm{CAG} \mathrm{Sun} 1 / \mathrm{sfGFP}$ mice induced a Cre-mediated recombination in activated $\mathrm{CreER}^{\mathrm{T} 2}$-expressing neurons, thus resulting in permanent GFP label on the nuclear membrane in the activated neurons. (B,C) Immunohistochemical GFP staining of coronal sections showing the DG and CA3 region of the hippocampus of female Arc-CreER ${ }^{\mathrm{T} 2} \times$ CAG-Sun1/sfGFP mice injected with ketamine (B) or (2R,6R)-HNK (C). Magnification $20 \times$, scale bar represents $200 \mu \mathrm{m}$. Insert pictures represented zoomed-in details. Ketamine and (2R,6R)-HNK did not significantly alter GFP-labelled Arc expression in DG (D) or CA3 (E). A trend towards significance is observed in CA3 (DG: one-way ANOVA, F $(2,5)=2.965, p=0.1416$; CA3: one-way ANOVA, F $(2,5)=5.566, p=0.0535)$. Arc expression is reflected in mean GFP intensity, which is displayed in mean gray values. Error bars represent \pm SEM. $N=2-3$ mice/group, 3 sections per animal, $n s=$ not significant. 


\subsubsection{Ketamine Increase BDNF Intensity in DG and CA3}

In order to confirm in females that ketamine and (2R,6R)-HNK increase $B d n f$ mRNa levels, we performed in situ hybridization (Figure 4A,B). The fluorescence mean intensity of $B d n f$ mRNA expression was measured for each condition. Statistical analysis demonstrated a significant difference in Bdnf level between groups in both DG (Figure 4C) and CA3 (Figure 4D). Bonferroni post-hoc test revealed a significant difference in $B d n f$ intensity between ketamine and saline treated females in both DG and CA3. Interestingly, in none of the hippocampal regions a significant difference was found between saline and (2R,6R)-HNK or ketamine and (2R,6R)-HNK (Figure 4).
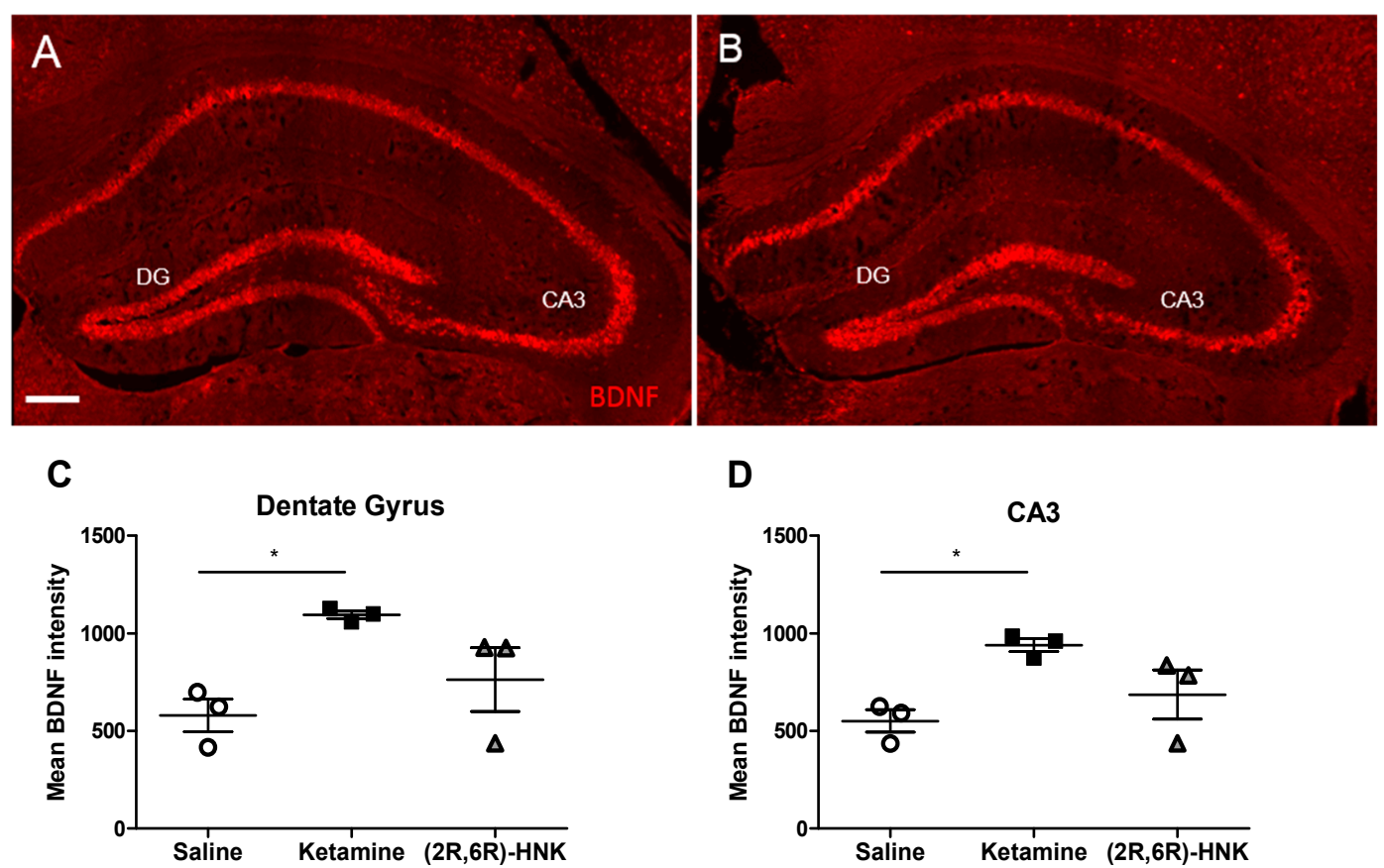

Figure 4. $B d n f$ mRNA expression in the hippocampal DG and CA3 after a single injection with ketamine or $(2 \mathrm{R}, 6 \mathrm{R})-\mathrm{HNK}$. (A,B) In situ hybridization with $B d n f$ probes on coronal sections showing the DG and $C A 3$ region of the hippocampus of female Arc-CreER ${ }^{\mathrm{T} 2} \times \mathrm{CAG}-\mathrm{Sun} 1 / \mathrm{sfGFP}$ mice injected with ketamine (A) or (2R,6R)-HNK (B). Magnification 20×, scale bar represents $200 \mu \mathrm{m}$. Ketamine but not (2R,6R)-HNK significantly increases Bdnf intensity in DG (C) and CA3 (D). (DG: one-way ANOVA, F $(2,6)=5.991, p=0.0371$, followed by Bonferroni post-test, ketamine vs. saline: $t=3.415, \mathrm{df}=6, p<0.05$. CA3: one-way ANOVA, F $(2,6)=5.754, p=0.0402$, followed by Bonferroni post-test, ketamine vs. saline: $t=3.341, \mathrm{df}=6, p<0.05)$. Mean $B d n f$ intensity is displayed in mean gray values, indicating $B d n f$ mRNA expression. Error bars represent \pm SEM. $N=3$ mice/group, 3 sections per animal. ${ }^{*} p<0.05$.

\section{Discussion}

Sex differences in neuropsychopharmacology have been overlooked for several years. Recently, rodent studies revealed that female and male are very diverse in several aspects. They showed different behavioral phenotype in multiple tests and they showed differences in pharmacokinetics and pharmacodynamic of several drugs. A very dramatic consequence for these differences is that a drug mainly developed and tested in male subjects, might show severe side effects in female subjects or not even show efficacy [27].

In the search for better antidepressant drugs, ketamine and its metabolite $(2 \mathrm{R}, 6 \mathrm{R})$-HNK represent a realistic new alternative [28]. However, the differences driven by sex in the antidepressant property of the two drugs are as yet fragmented. In this study we performed a baseline characterization on the sex differences of the Arc-CreER ${ }^{\mathrm{T} 2} \times \mathrm{CAG}-\mathrm{Sun} 1 / \mathrm{sfGFP}$ mice treated with ketamine and (2R,6R)-HNK. Using a test battery previously established by our group [26], we found that both ketamine and 
(2R,6R)-HNK did not affect memory, anxiety-like behavior, sociability, and locomotion $24 \mathrm{~h}$ after administration but that sex significantly influenced the mouse performance in the sociability and anxiety tests [8,29-32]. That neither ketamine nor (2R,6R)-HNK influenced memory, sociability, nor locomotion is in line with previous findings. However contrary to our results ketamine has been previously reported to act as an anxiolytic $[9,33]$. An explanation for this divergence could be found in the dose at which ketamine was used. Previous research suggested that the anxiolytic effect of ketamine is dose dependent, with low doses leading to anxiolytic effects, but high doses increasing anxiety symptoms [34]. With a dose of $10 \mathrm{mg} \mathrm{kg}^{-1}$ the anxiolytic effect was detectable [33], thus the dose used in the current study may have been too high to elicit anxiolytic effects. On the other hand, the evidence for an anxiolytic effect of ketamine seems to be rare and ambiguous, as studies found anxiolytic effects [9,33], but others found anxiogenic effects [35].

Interestingly, in our study we found a significant sex difference in the sociability test and LD box test showing that these behaviors might be affected by sex.

As a classical tool to study antidepressant-like effects of drugs, we subjected male and female mice to the FST. We found a sex $\times$ treatment interaction. Indeed, in the FST ketamine and $(2 R, 6 R)-H N K$ had opposite effects in female and male mice. Moreover, we showed that ketamine and (2R,6R)-HNK treated male mice differed in the FST, with ketamine treated mice being less immobile compared to $(2 \mathrm{R}, 6 \mathrm{R})-\mathrm{HNK}$ treated mice. However, in our study we could not find a statistical difference between ketamine treated male mice and saline treated ones. The absence of this difference may have been caused by the absence of a stressor in our experimental procedure [10]. It was shown that stressed animals administered with ketamine at the same dose used in this study exhibited antidepressant-like effects, whereas unstressed animals did not. This highlights the importance of the implementation of a stressor beforehand for ketamine and (2R,6R)-HNK to elicit their antidepressant-like effect. Moreover, in the rodent literature there is no consensus yet on the antidepressant-like effects of ketamine. Additionally, these effects seem to be dependent on the dose, species and strain, test, stressor, and the sex of the experimenter as highlighted in a recent review [36]. Therefore, we once more highlight that the interpretation of our findings must remain limited to the sex of the tested animals for each test/measurement and to the specific doses that we applied in our studies.

Since we demonstrated an opposite effect in female and male mice in the FST, we wanted to understand whether at molecular level females would show a different pattern compared to what is reported in the literature for male mice. Therefore, we focused on BDNF which is widely considered as a main downstream mediator in the effect of ketamine [18]

By using the elegant design of the Arc-CreER ${ }^{\mathrm{T} 2} \times$ CAG-Sun1/sfGFP mice, we were able to, in the presence of TM, permanently label the neurons that upon activation underwent Cre-dependent recombination. Firstly, we analyzed the GFP intensity level in all experimental groups and hypothesized an increase of GFP intensity dependent on the activation on the Arc promoter (see Figure 3A). Previously, studies indeed reported that ketamine induced Arc expression [25,37]. Although no significant increases in GFP intensity were found in either the DG or the CA3, we found a strong trend towards significance in the CA3 region of the hippocampus. However, a recent study using the same line lacked in finding a significant increase in the number of activated cells after ketamine treatment in a fear conditioning experiment [38], but the authors were able to detect an increase in the colocalization between activated neurons and c-fos (the protein of interest).

Surprisingly, in our study, we found a significant increase in Bdnf mRNA expression in the DG and CA3 region of the dorsal hippocampus of females injected with ketamine compared to control. These findings suggested a mismatch between behavioral and molecular findings. Ketamine revealed different effects in the FST in male and female but led to an increase in Bdnf level in females (Figure 4) as previously reported for males [18]. Strikingly, no significant increase in $B d n f$ expression was found in the dorsal hippocampus of females injected with $(2 \mathrm{R}, 6 \mathrm{R})-\mathrm{HNK}$. These findings are supported by a recent study that identified the medial prefrontal cortex as the key region for the actions of (2R,6R)-HNK [23]. The present results, together with these previous findings, suggest that $(2 \mathrm{R}, 6 \mathrm{R})-\mathrm{HNK}$ 
acts through a different neuroanatomical network than ketamine in female mice. Unfortunately, as the main limitation of this study, we were not able to stain BDNF protein in the GFP positive activated neurons. BDNF antibodies show indeed discontinuity among batches and lack of reproducibility in staining. Moreover, we performed the molecular study only in female mice, thus a comparison with male mice is only possible based on the literature. Therefore, further studies on a different cohort of animals might establish an increase of BDNF in the subset of GFP positive activated neurons both in male and female mice.

\section{Materials and Methods}

\subsection{Animals}

Heterozygous Arc-CreER ${ }^{\mathrm{T} 2}$ mice (Jax \#022357) $[24,37,39]$ and homozygous R26-CAG-LSLSun1/sfGFP-myc mice (Jax \#021039) [40] were obtained from The Jackson Laboratory (USA) and were bred and crossed in-house to generate heterozygous Arc-CreER ${ }^{\mathrm{T} 2} \times$ CAG-Sun1/sfGFP mice. Both male and female offspring heterozygous for both the reporter and CreER ${ }^{\mathrm{T} 2}$ gene were used in the experiments. At the age of 6 weeks, mice left the breeding area and entered our mouse behavioral unit. Mice were allowed to habituate to the new environment for at least one week. Mice were single-housed one week prior to the experiment. After entering our mouse behavioral unit, male and female mice were always housed in separate rooms.

Mice were housed in a room with $24 \pm 1{ }^{\circ} \mathrm{C}$ and $40 \%$ humidity and maintained on a 12:12 $\mathrm{h}$ light/dark cycle with lights on at 7 am during the whole experiment. All animals were provided with food and water ad libitum. All experiments were carried out in accordance with the European Community's Council Directive of 22 September 2010 (2010/63EU) and approved by the local authorities (Animal Protection Committee of the State Government, Landesuntersuchungsamt Rheinland-Pfalz, Koblenz, Germany).

\subsection{Compounds}

Tamoxifen (Cat\#T5648, Sigma, St. Louis, MO, USA) was dissolved in $99.8 \%$ ethanol to reach a concentration of $100 \mathrm{mg} \mathrm{mL}^{-1}$. The tamoxifen/ethanol mixture was added to corn oil (Cat\#C8267, Sigma, St. Louis, MO, USA) to reach a final concentration of $10 \mathrm{mg} \mathrm{mL}^{-1}$ and incubated for $15 \mathrm{~min}$ at $37^{\circ} \mathrm{C}$. To induce Cre recombination, the tamoxifen mixture was administered by a single intraperitoneal injection (150 mg kg$\left.{ }^{-1}\right)$ followed by a single intraperitoneal injection with one of the compounds, saline or ketamine or (2R,6R)-HNK. Tamoxifen was injected to make active CreERT2-expressing cells undergo Cre-mediated recombination, resulting in permanent expression of GFP. Intensity of the GFP protein was measured after immunohistochemistry (see Section 4.5). Racemic ketamine (Inresa Arzneimittel GmbH, Freiburg, Germany) and (2R,6R)-HNK (Tocris Bioscence, Bristol, UK) were dissolved in $\mathrm{NaCl}$ 0.9\% (Saline, Braun, Melsungen, Germany) following manufactural descriptions. A single intraperitoneal injection of either ketamine $\left(30 \mathrm{mg} \mathrm{kg}^{-1}\right)$ [41], (2R,6R)-HNK $\left(10 \mathrm{mg} \mathrm{kg}^{-1}\right)$ [8], or saline $(100 \mu \mathrm{L})$ was administered $24 \mathrm{~h}$ prior to behavioral testing.

\subsection{Behavioral Procedure}

Mice were handled for 2 min per day on the five days preceding an injection with one of the compounds. Mice were randomly assigned to a treatment group. Twenty-four hours prior to the behavioral battery or forced swim test (FST), mice obtained a single intraperitoneal injection with saline, ketamine, or $(2 \mathrm{R}, 6 \mathrm{R})-\mathrm{HNK}$. Test battery was performed in a sound attenuated environment. Male and female mice were tested in separate rooms. Behavioral testing was conducted in the light phase (9-12 a.m.) under dimmed light conditions ( $39 \pm 2$ lux), except during the light dark box (600 \pm 2 lux). Animals were randomized and experimenters were blinded for the treatment conditions during testing, scoring, and analyses. 


\subsubsection{Behavioral Battery}

The behavioral battery enables a brief $(3 \mathrm{~h})$ and condensed testing of multiple important behavioral domains. The present behavioral battery was slightly modified from the behavioral battery described by Jene and colleagues [26]. Additional to the spontaneous alternations, spatial object recognition task (SORT), novel object recognition task (NORT), sociability test, light-dark box, and bedding preference task, the present behavioral battery included an open field (OF) to measure locomotor activity for $10 \mathrm{~min}$ which was conducted in an OF arena $(45 \times 45 \times 41 \mathrm{~cm})$ prior to the NORT (Figure 5). During inter-test intervals (i.t.i.), lasting $15 \mathrm{~min}$, set-ups were cleaned with $5 \%$ ethanol.

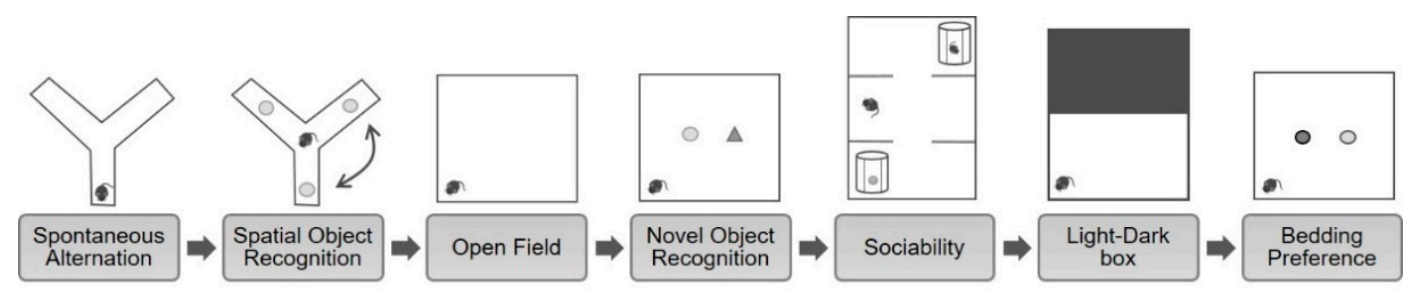

Figure 5. Schematic of the behavioral battery. This battery comprises a comprehensive behavioral battery to investigate multiple behavioral domains. In chronological order the battery consists of spontaneous alternation behavior in the Y-maze (working memory), spatial object recognition task (spatial memory), open field (locomotor activity), novel object recognition task (episodic memory), sociability test (social preference), light-dark box (anxiety-like behavior), and bedding preference task (olfaction) with $15 \mathrm{~min}$ of inter-test intervals.

\subsubsection{Forced Swim Test (FST)}

Depressive-like behavior was assessed in the FST in a separate batch of animals. Each animal was placed in a glass cylinder (height: $24 \mathrm{~cm}$; diameter: $13 \mathrm{~cm}$ ) filled with water (depth: $15 \mathrm{~cm}$; temperature: $21 \pm 1{ }^{\circ} \mathrm{C}$ ) for $5 \mathrm{~min}$ while behavior was video recorded from the side. Videos were manually scored on swimming, climbing, and floating behavior. Floating is represented as immobility time (s). Swimming and climbing behavior are scored together as mobility time (s). Water temperature was measured and adjusted following each test.

\subsection{Tissue Collection}

Drug-naïve female mice received two intraperitoneal injections with tamoxifen and with either saline, ketamine, or $(2 \mathrm{R}, 6 \mathrm{R})$-HNK and were maintained in an isolated, sound-attenuated room (temperature: $22^{\circ} \mathrm{C}$ ). Three days following the injections, mice were anesthetized with pentobarbital (100 $\mathrm{mg} \mathrm{kg}^{-1}$ bodyweight) and buprenorphine $\left(0.1 \mathrm{mg} \mathrm{kg}^{-1}\right.$ bodyweight) and manually perfused with 10mL cold PBS1x (Thermofisher Scientific, USA) followed by $10 \mathrm{~mL}$ cold $4 \%$ paraformaldehyde (PFA, Santa Cruz Biotechnology, USA). Brains were dissected and post-fixed in $4 \%$ PFA overnight at $4{ }^{\circ} \mathrm{C}$, treated with $30 \%$ sucrose for two days and stored at $-80^{\circ} \mathrm{C}$ until further processing.

\subsection{Immunohistochemistry}

Female brains were sectioned at $30 \mu \mathrm{m}$ thickness in coronal plane using a Kryostat HM 560 Microm and stored in cryoprotectant (20\% glycerol (87\%), 30\% ethylene glycerol, 50\% PBS1x) until use. Immunohistochemistry was performed on $30 \mu \mathrm{m}$ dorsal hippocampal sections of the female brain. Sections were rinsed three times with PBS1x for $5 \mathrm{~min}$ to remove cryoprotectant, followed by a wash with PBS1x/0.2\%Triton-X100(TX) for 5 min. Sections were blocked with $4 \%$ goat serum for $15 \mathrm{~min}$ and incubated with a primary antibody against GFP (Aveslabs cat\#GFP-1020, chicken, 1:500) overnight at $4{ }^{\circ} \mathrm{C}$. Sections were rinsed three times with $\mathrm{PBS} 1 \mathrm{x} / 0.2 \% \mathrm{TX}$ for $5 \mathrm{~min}$ to remove the antibody and were incubated with the secondary antibody goat anti-chicken fluorescein labelled 488 (Aveslabs cat\#F-1005, 1:1000) for $1 \mathrm{~h}$ in the dark. Remaining antibody was removed by rinsing two times with PBS1x for 
5 min, followed by incubation with DAPI (1:5000 from $10 \mathrm{mg} \mathrm{mL}^{-1}$ stock diluted in PBS1x) for $5 \mathrm{~min}$. Sections were rinsed with PBS1x before mounting with DAKO fluorescence mounting medium.

\subsection{In Situ Hybridization}

\subsubsection{Bdnf RNA Probe Synthesis}

A plasmid (pT7T3D-Pacl, 2897kb, TransOMIC Technologies, Huntsville, AL, USA) containing cDNA of BDNF (NM_007540) and promoter elements for T3 and T7 RNA polymerases was used. The plasmid was cut using restriction enzymes (HINDIII for sense, XLOI for anti-sense) and subsequently linearized. The transcription of this linearized plasmid resulted in single-strand RNA probes; the sense and the anti-sense probe. The BDNF antisense probe was used during the experiment, the sense probe served as control. The incubation with the sense riboprobe did not show any signal.

\subsubsection{Digoxigenin (DIG) Labelling}

Single-stranded RNA probes were labelled with DIG-nucleotides following the DIG RNA Labeling Mix procedure (Roche Diagnostics, Mannheim, Germany). The labelled RNA probes were cleaned up using the RNeasy Mini Kit (Qiagen, Hilden, Germany). Quality and quantity of the DIG-labelled probes were analyzed by electrophoresis of a $1 \%$ agarose gel with ethidium bromide staining.

\subsubsection{In Situ Hybridization}

For the same female brains, dorsal hippocampus was sectioned at $18 \mu \mathrm{m}$ thickness in coronal plane and mounted on microscope slides (Superfrost Plus, Thermo Fisher Scientific, Dreieich, Germany). Slides were fixed in $4 \%$ PFA at $4{ }^{\circ} \mathrm{C}$ for $20 \mathrm{~min}$ and rinsed two times with PBS1x for $5 \mathrm{~min}$. Slides were incubated with $0.2 \mathrm{M} \mathrm{HCl}$ for $8 \mathrm{~min}$ and rinsed again with PBS1x for $2 \mathrm{~min}$. Treatment with $0.4 \mathrm{U} / \mathrm{mL}$ proteinase $\mathrm{K}$ in TE buffer (50 $\mathrm{mM}$ Tris/5 $\mathrm{mM}$ EDTA) was performed for $10 \mathrm{~min}$. Slides were rinsed with PBS1x for $5 \mathrm{~min}$ and soaked in $4 \%$ PFA at $4{ }^{\circ} \mathrm{C}$ for $20 \mathrm{~min}$. Incubation with acetic anhydride $(2 \times 600 \mu \mathrm{L})$ in $0.1 \mathrm{M}$ triethanolamine was performed for $10 \mathrm{~min}$ followed by a wash with PBS1x for $5 \mathrm{~min}$. Sections on the slides were dehydrated with saline for $5 \mathrm{~min}$ followed by an ethanol series of $30 \%, 50 \%, 70 \%, 80 \%, 95 \%$, and two times $100 \%$. Antisense ( $600 \mathrm{ng}$ probe $\mathrm{mL}^{-1}$ ) and sense probes were dissolved in hybridization mix ( $50 \%$ formamide, $20 \mathrm{mM}$ Tris- $\mathrm{HCl} \mathrm{pH}$ 8.0, 0.3 NaCl, $5 \mathrm{mM}$ EDTA pH8.0, $10 \%$ dextran sulfate, $0.02 \%$ ficol, $0.02 \%$ Polyvinylpyrrolidone, $0.02 \%$ BSA, $0.5 \mathrm{mg} / \mathrm{mL}$ tRNA (Sigma 10109517001), $0.2 \mathrm{mg} / \mathrm{mL}$ Carrier DNA, 200mM DTT) and heated for $5 \mathrm{~min}$ at $37^{\circ} \mathrm{C}$. Slides were incubated with the antisense or sense probe solutions overnight at $55 \pm 1{ }^{\circ} \mathrm{C}$. To prevent evaporation of the hybridization buffer, sections were covered with coverslips. After hybridization, slides were dipped in $5 \times$ saline-sodium citrate buffer (SSC) to detach the coverslips and were placed directly in $2 \times$ SSC containing $50 \%$ formamide for $30 \mathrm{~min}$ at $62{ }^{\circ} \mathrm{C}$ to remove remaining probe and hybridization buffer. Slides were washed with $1 \times$ SSC in $50 \%$ formamide for $30 \mathrm{~min}$ at $62^{\circ} \mathrm{C}$ and finally with $0.1 \times$ SSC for $30 \mathrm{~min}$ at $62{ }^{\circ} \mathrm{C}$ to remove non-specific DNA/RNA hybridization. Slides were incubated with $4 \%$ sheep serum for $1 \mathrm{~h}$. For antibody administration, slides were switched to Shandon Cassettes. Slides were rinsed two times with Tris-NaCl-Tween (TNT) buffer for $2 \mathrm{~min}$ at $30^{\circ} \mathrm{C}$, followed by a wash with PE buffer (Qiagen, Hilden, Germany) for $30 \mathrm{~min}$ at $30^{\circ} \mathrm{C}$ and incubated with anti-DIG fragments (Roche Diagnostics, diluted 1:1000 in PE buffer) for $2 \mathrm{~h}$ at $30^{\circ} \mathrm{C}$. Slides were rinsed three times with TNT for $2 \mathrm{~min}$ at $30^{\circ} \mathrm{C}$ and incubated with tyramine $\mathrm{CY} 3$ diluted (1:50) in amplification diluent (TSA Cyanine 3 Reagent Kit, Perkin Elmer, Germany) for 15 min at $30^{\circ} \mathrm{C}$ in the dark. Slides were rinsed with PBS1x for $2 \mathrm{~min}$ and incubated with DAPI (1:5000) for $5 \mathrm{~min}$. Slides were rinsed three times with PBS1x for $2 \mathrm{~min}$, rinsed in autoclaved $\mathrm{H} 2 \mathrm{O}$ and mounted using DAKO fluorescence mounting medium. 


\subsection{Microscopy}

For each brain three dorsal hippocampal sections were analyzed. Tile scans of the whole hippocampus were made using a Leica AF7000 wide field microscope equipped with a Hamamatsu-Flash4-USB3-101292 camera and LAS X software (Institute for Molecular Biology, Mainz, Germany). Images were acquired with a HC PL FLUOTAR L 20×/0.40 objective lens using the same settings for each section. Images visualizing GFP fluorescence were acquired using an L5 filter, DAPI was imaged using an A4 filter, and Bdnf expression (Cy3) was imaged using an N3 filter. Single images were merged to obtain a complete tile scan. Tile scans were used to enable DG and CA3 analysis in Fiji (ImageJ, Bethesda, MD, USA).

\subsection{Intensity Quantification}

Bilateral DG and CA3 regions of the hippocampus were selected and the mean GFP and BDNF intensity were quantified using the Fiji software. The experimenter was blind to treatment conditions during quantification. Intensity levels were averaged per animal and subsequently compared between treatment groups. One ketamine-injected animal was excluded from further GFP analysis, due to experimental errors.

\subsection{Statistical Analysis}

Statistical analysis was performed with the Prism version 5 software (Graphpad software Inc., San Diego, CA, USA). All data was tested for normality with the D'Agostino and Pearson omnibus normality test. Behavioral battery data was first analyzed using a one-sample $t$-test against chance level. Battery and FST data were analyzed using a one-way ANOVA, only spontaneous alternation was analyzed using a two-way ANOVA, followed by a Bonferroni post-test. Effect of both sex and treatment on battery and FST data was evaluated using a two-way ANOVA followed by a Bonferroni post-test when applicable. Intensity values were analyzed using a one-way ANOVA followed by a Bonferroni post-test. All data are expressed as means \pm SEM and were considered significant at $p<0.05$.

Author Contributions: Conceptualization, D.P.H., M.B.M. and G.T.; methodology D.H.P., R.M.M., F.R., G.T.; data analysis, D.H.P., R.M.M.; G.T.; writing-review and editing, D.H.P., R.M.M.; F.R., M.B.M., B.L., G.T.; funding acquisition, M.B.M., B.L., G.T. All authors have read and agreed to the published version of the manuscript.

Funding: D.P.H. is supported by the Mainz Research School of Translational Biomedicine (TransMed) with a MD/PhD-fellowship. G.T. is supported by a 2014 NARSAD Young Investigator Grant from the Brain \& Behaviour Research Foundation and the Danish Council for Independent Research grant number DFF-5053-00103. B.L. and M.B.M. are supported by the German Research Foundation (DFG) within the Collaborative Research Centre 1193 (CRC1193, https://crc1193.de/) and by the Boehringer Ingelheim Foundation grant number BIS09.

Acknowledgments: We would like to thank Sandra Ritz and the Core Facility Microscopy at the Institute of Molecular Biology (IMB, Mainz, Germany) for excellent support using microscopy and image analysis. We would like to thank Andrea Conrad, Ruth Jelinek, and Julia Deuster for excellent technical support, and Diego Pascual Cuadrado for technical support with the TRAP mice.

Conflicts of Interest: The authors declare no conflict of interest 


\section{Appendix A}

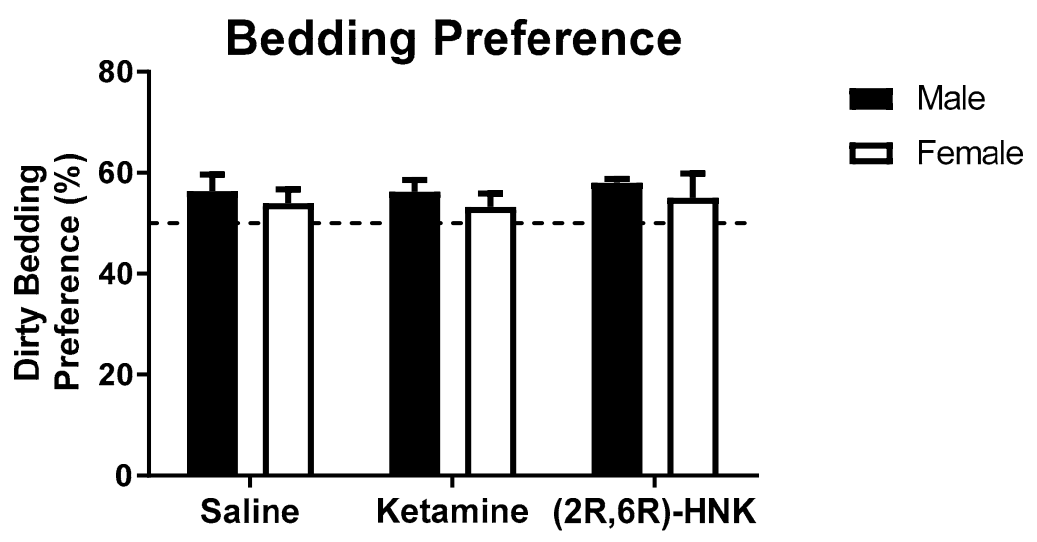

Figure A1. Ketamine and (2R,6R)-HNK did not affect olfaction of Arc-CreER ${ }^{\mathrm{T} 2} \times \mathrm{CAG}$-Sun1/sfGFP mice. Olfaction in the bedding preference. Preference for dirty bedding was measured and is presented in percentage. Dotted line represents chance level. Male $n=22$, female $n=39$. All behaviors are expressed as mean percentage and error bars represent SEM. No effect of treatment or sex (two-way ANOVA).

\section{References}

1. Patel, V.; Chisholm, D.; Parikh, R.; Charlson, F.J.; Degenhardt, L.; Dua, T.; Ferrari, A.J.; Hyman, S.; Laxminarayan, R.; Levin, C.; et al. Addressing the burden of mental, neurological, and substance use disorders: Key messages from Disease Control Priorities, 3rd edition. Lancet 2016, 387, 1672-1685. [CrossRef]

2. Machado-Vieira, R.; Baumann, J.; Wheeler-Castillo, C.; Latov, D.; Henter, I.D.; Salvadore, G.; Zarate, C.A. The Timing of Antidepressant Effects: A Comparison of Diverse Pharmacological and Somatic Treatments. Pharmaceuticals 2010, 3, 19-41. [CrossRef] [PubMed]

3. Duman, R.S.; Aghajanian, G.K.; Sanacora, G.; Krystal, J.H. Synaptic plasticity and depression: New insights from stress and rapid-acting antidepressants. Nat. Med. 2016, 22, 238-249. [CrossRef] [PubMed]

4. Berman, R.M.; Cappiello, A.; Anand, A.; Oren, D.A.; Heninger, G.R.; Charney, D.S.; Krystal, J.H. Antidepressant effects of ketamine in depressed patients. Biol. Psychiatry 2000, 47, 351-354. [CrossRef]

5. Zarate, C.A., Jr.; Singh, J.B.; Carlson, P.J.; Brutsche, N.E.; Ameli, R.; Luckenbaugh, D.A.; Charney, D.S.; Manji, H.K. A randomized trial of an N-methyl-D-aspartate antagonist in treatment-resistant major depression. Arch. Gen. Psychiatry 2006, 63, 856-864. [CrossRef]

6. Short, B.; Fong, J.; Galvez, V.; Shelker, W.; Loo, C.K. Side-effects associated with ketamine use in depression: A systematic review. Lancet Psychiatry 2018, 5, 65-78. [CrossRef]

7. Daly, E.J.; Trivedi, M.H.; Janik, A.; Li, H.; Zhang, Y.; Li, X.; Lane, R.; Lim, P.; Duca, A.R.; Hough, D.; et al. Efficacy of Esketamine Nasal Spray Plus Oral Antidepressant Treatment for Relapse Prevention in Patients With Treatment-Resistant Depression: A Randomized Clinical Trial. JAMA Psychiatry 2019, 76, 893-903. [CrossRef]

8. Zanos, P.; Moaddel, R.; Morris, P.J.; Georgiou, P.; Fischell, J.; Elmer, G.I.; Alkondon, M.; Yuan, P.; Pribut, H.J.; Singh, N.S.; et al. NMDAR inhibition-independent antidepressant actions of ketamine metabolites. Nature 2016, 533, 481-486. [CrossRef]

9. Zhang, L.M.; Zhou, W.W.; Ji, Y.J.; Li, Y.; Zhao, N.; Chen, H.X.; Xue, R.; Mei, X.G.; Zhang, Y.Z.; Wang, H.L.; et al. Anxiolytic effects of ketamine in animal models of posttraumatic stress disorder. Psychopharmacology (Berl) 2015, 232, 663-672. [CrossRef]

10. Fitzgerald, P.J.; Yen, J.Y.; Watson, B.O. Stress-sensitive antidepressant-like effects of ketamine in the mouse forced swim test. PLoS ONE 2019, 14, e0215554. [CrossRef]

11. Ding, R.; Li, Y.; Du, A.; Yu, H.; He, B.; Shen, R.; Zhou, J.; Li, L.; Cui, W.; Zhang, G.; et al. Changes in hippocampal AMPA receptors and cognitive impairments in chronic ketamine addiction models: Another understanding of ketamine CNS toxicity. Sci. Rep. 2016, 6, 38771. [CrossRef]

12. Dossat, A.M.; Wright, K.N.; Strong, C.E.; Kabbaj, M. Behavioral and biochemical sensitivity to low doses of ketamine: Influence of estrous cycle in C57BL/6 mice. Neuropharmacology 2018, 130, 30-41. [CrossRef] [PubMed] 
13. Franceschelli, A.; Sens, J.; Herchick, S.; Thelen, C.; Pitychoutis, P.M. Sex differences in the rapid and the sustained antidepressant-like effects of ketamine in stress-naive and "depressed" mice exposed to chronic mild stress. Neuroscience 2015, 290, 49-60. [CrossRef] [PubMed]

14. Saland, S.K.; Kabbaj, M. Sex Differences in the Pharmacokinetics of Low-dose Ketamine in Plasma and Brain of Male and Female Rats. J. Pharmacol. Exp. Ther. 2018, 367, 393-404. [CrossRef] [PubMed]

15. Herzog, D.P.; Wegener, G.; Lieb, K.; Muller, M.B.; Treccani, G. Decoding the Mechanism of Action of Rapid-Acting Antidepressant Treatment Strategies: Does Gender Matter? Int. J. Mol. Sci. 2019, $20,949$. [CrossRef]

16. Li, N.; Lee, B.; Liu, R.J.; Banasr, M.; Dwyer, J.M.; Iwata, M.; Li, X.Y.; Aghajanian, G.; Duman, R.S. mTOR-dependent synapse formation underlies the rapid antidepressant effects of NMDA antagonists. Science 2010, 329, 959-964. [CrossRef]

17. Li, N.; Liu, R.J.; Dwyer, J.M.; Banasr, M.; Lee, B.; Son, H.; Li, X.Y.; Aghajanian, G.; Duman, R.S. Glutamate $\mathrm{N}$-methyl-D-aspartate receptor antagonists rapidly reverse behavioral and synaptic deficits caused by chronic stress exposure. Biol. Psychiatry 2011, 69, 754-761. [CrossRef]

18. Autry, A.E.; Adachi, M.; Nosyreva, E.; Na, E.S.; Los, M.F.; Cheng, P.F.; Kavalali, E.T.; Monteggia, L.M. NMDA receptor blockade at rest triggers rapid behavioural antidepressant responses. Nature 2011, 475, 91-95. [CrossRef]

19. Bjorkholm, C.; Monteggia, L.M. BDNF-A key transducer of antidepressant effects. Neuropharmacology 2016, 102, 72-79. [CrossRef]

20. Deyama, S.; Duman, R.S. Neurotrophic mechanisms underlying the rapid and sustained antidepressant actions of ketamine. Pharmacol. Biochem. Behav. 2020, 188, 172837. [CrossRef]

21. Cavalleri, L.; Merlo Pich, E.; Millan, M.J.; Chiamulera, C.; Kunath, T.; Spano, P.F.; Collo, G. Ketamine enhances structural plasticity in mouse mesencephalic and human iPSC-derived dopaminergic neurons via AMPAR-driven BDNF and mTOR signaling. Mol. Psychiatry 2018, 23, 812-823. [CrossRef] [PubMed]

22. Yao, N.; Skiteva, O.; Zhang, X.; Svenningsson, P.; Chergui, K. Ketamine and its metabolite (2R,6R)-hydroxynorketamine induce lasting alterations in glutamatergic synaptic plasticity in the mesolimbic circuit. Mol. Psychiatry 2018, 23, 2066-2077. [CrossRef] [PubMed]

23. Fukumoto, K.; Fogaca, M.V.; Liu, R.J.; Duman, C.; Kato, T.; Li, X.Y.; Duman, R.S. Activity-dependent brain-derived neurotrophic factor signaling is required for the antidepressant actions of (2R,6R)-hydroxynorketamine. Proc. Natl. Acad. Sci. USA 2019, 116, 297-302. [CrossRef] [PubMed]

24. Guenthner, C.J.; Miyamichi, K.; Yang, H.H.; Heller, H.C.; Luo, L. Permanent genetic access to transiently active neurons via TRAP: Targeted recombination in active populations. Neuron 2013, 78, 773-784. [CrossRef] [PubMed]

25. Choi, M.; Lee, S.H.; Wang, S.E.; Ko, S.Y.; Song, M.; Choi, J.S.; Kim, Y.S.; Duman, R.S.; Son, H. Ketamine produces antidepressant-like effects through phosphorylation-dependent nuclear export of histone deacetylase 5 (HDAC5) in rats. Proc. Natl. Acad. Sci. USA 2015, 112, 15755-15760. [CrossRef] [PubMed]

26. Jene, T.; Gassen, N.C.; Opitz, V.; Endres, K.; Muller, M.B.; van der Kooij, M.A. Temporal profiling of an acute stress-induced behavioral phenotype in mice and role of hippocampal DRR1. Psychoneuroendocrinology 2018, 91, 149-158. [CrossRef]

27. Parekh, A.; Fadiran, E.O.; Uhl, K.; Throckmorton, D.C. Adverse effects in women: Implications for drug development and regulatory policies. Expert Rev. Clin. Pharmacol. 2011, 4, 453-466. [CrossRef]

28. Witkin, J.M.; Martin, A.E.; Golani, L.K.; Xu, N.Z.; Smith, J.L. Rapid-acting antidepressants. Adv. Pharmacol. 2019, 86, 47-96.

29. Engin, E.; Treit, D. Anxiolytic and antidepressant actions of somatostatin: The role of sst2 and sst3 receptors. Psychopharmacology (Berl) 2009, 206, 281-289. [CrossRef]

30. Rezvani, A.H. Involvement of the NMDA System in Learning and Memory. In Animal Models of Cognitive Impairment; Levin, E.D., Buccafusco, J.J., Eds.; CRC Press: Boca Raton, FL, USA, 2006.

31. Hanks, A.N.; Dlugolenski, K.; Hughes, Z.A.; Seymour, P.A.; Majchrzak, M.J. Pharmacological disruption of mouse social approach behavior: Relevance to negative symptoms of schizophrenia. Behav. Brain Res. 2013, 252, 405-414. [CrossRef]

32. Ribeiro, P.O.; Rodrigues, P.C.; Valentim, A.M.; Antunes, L.M. A single intraperitoneal injection of ketamine does not affect spatial working, reference memory or neurodegeneration in adult mice: An animal study. Eur. J. Anaesthesiol. 2013, 30, 618-626. [CrossRef] [PubMed] 
33. Fraga, D.B.; Olescowicz, G.; Moretti, M.; Siteneski, A.; Tavares, M.K.; Azevedo, D.; Colla, A.R.S.; Rodrigues, A.L.S. Anxiolytic effects of ascorbic acid and ketamine in mice. J. Psychiatr. Res. 2018, 100, 16-23. [CrossRef] [PubMed]

34. Krystal, J.H.; Karper, L.P.; Seibyl, J.P.; Freeman, G.K.; Delaney, R.; Bremner, J.D.; Heninger, G.R.; Bowers, M.B., Jr.; Charney, D.S. Subanesthetic effects of the noncompetitive NMDA antagonist, ketamine, in humans. Psychotomimetic, perceptual, cognitive, and neuroendocrine responses. Arch. Gen. Psychiatry 1994, 51, 199-214. [CrossRef] [PubMed]

35. Silvestre, M.A.; Lucena, J.E.; Roxas, R., Jr.; Evangelista, E.S.; Ostrea, E.M., Jr. Effects of timing, dosage, and duration of morphine intake during pregnancy on the amount of morphine in meconium in a rat model. Biol. Neonate 1997, 72, 112-117. [CrossRef] [PubMed]

36. Polis, A.J.; Fitzgerald, P.J.; Hale, P.J.; Watson, B.O. Rodent ketamine depression-related research: Finding patterns in a literature of variability. Behav. Brain Res. 2019, 376, 112153. [CrossRef] [PubMed]

37. Denny, C.A.; Kheirbek, M.A.; Alba, E.L.; Tanaka, K.F.; Brachman, R.A.; Laughman, K.B.; Tomm, N.K.; Turi, G.F.; Losonczy, A.; Hen, R. Hippocampal memory traces are differentially modulated by experience, time, and adult neurogenesis. Neuron 2014, 83, 189-201. [CrossRef]

38. Mastrodonato, A.; Martinez, R.; Pavlova, I.P.; LaGamma, C.T.; Brachman, R.A.; Robison, A.J.; Denny, C.A. Ventral CA3 Activation Mediates Prophylactic Ketamine Efficacy Against Stress-Induced Depressive-like Behavior. Biol. Psychiatry 2018, 84, 846-856. [CrossRef]

39. Root, C.M.; Denny, C.A.; Hen, R.; Axel, R. The participation of cortical amygdala in innate, odour-driven behaviour. Nature 2014, 515, 269-273. [CrossRef]

40. Mo, A.; Mukamel, E.A.; Davis, F.P.; Luo, C.; Henry, G.L.; Picard, S.; Urich, M.A.; Nery, J.R.; Sejnowski, T.J.; Lister, R.; et al. Epigenomic Signatures of Neuronal Diversity in the Mammalian Brain. Neuron 2015, 86, 1369-1384. [CrossRef]

41. Brachman, R.A.; McGowan, J.C.; Perusini, J.N.; Lim, S.C.; Pham, T.H.; Faye, C.; Gardier, A.M.; Mendez-David, I.; David, D.J.; Hen, R.; et al. Ketamine as a Prophylactic Against Stress-Induced Depressive-like Behavior. Biol. Psychiatry 2016, 79, 776-786. [CrossRef] 